\title{
A Direct Down-conversion System Using Sub- harmonic Mixer with Microstrip Lines
}

\author{
Yu-Chang Lin ${ }^{1, *}$, Wen $\mathrm{Ko}^{2}$, Ming-Wei Hsu ${ }^{3}$ and Man-Long $\mathrm{Her}^{4}$ \\ ${ }^{1} \mathrm{Ph} . D$. Program in Electrical and Communications Engineering, Feng-Chia University, 100, Wen-Hua Rd., Taichung, 40724, \\ Taiwan R.O.C. \\ ${ }^{2}$ Department of Electronic Engineering, National Chin-Yi University of Technology, 57, Sec. 2, Zhongshan Rd., Taiping Dist., \\ Taichung 41170, Taiwan R.O.C. \\ ${ }^{3}$ Department of Electronics Engineering, Nan Kai University of Technology, 568, Zhongzheng Rd., Caotun Township, Nantou \\ County 54243, Taiwan R.O.C. \\ ${ }^{4}$ Department of Communications Engineering, Feng-Chia University, 100, Wen-Hua Rd., Taichung, 40724, Taiwan R.O.C. \\ ${ }^{*}$ Corresponding author
}

\begin{abstract}
The purpose of this paper is to design a direct downconversion system using sub-harmonic mixer with microstrip lines, it is also called a zero intermediate frequency system. By utilizing the direct conversion configuration, it can not only avoid to use an additional voltage controlled oscillator and phaselocked loop which resulted from twice down conversions so as to simplify the configuration and reduce cost, but also suppress the mirror image frequency, and thus to diminish non-linear conversion distortion and to increase the receive dynamic range. The LO-RF and LO-IF isolations of sub-harmonic mixer with microstrip lines are greater than $60 \mathrm{~dB}$, and the conversion loss is about $14 \mathrm{~dB}$; the phase noise of VCO with spiral-shaped defected ground structure (DGS) is $-96 \mathrm{dBc} / \mathrm{Hz}$ at $10 \mathrm{kHz}$. We have succeeded to develop a direct down-conversion system.
\end{abstract}

Keywords- direct down-conversion system; sub-harmonic mixer; zero intermediate frequency; Voltage Controlled Oscillator (VCO); Phase-locked Loop (PLL); Defected Ground Structure (DGS); microstrip line

\section{INTRODUCTION}

Recently, owing to the demand for simple, light weight and low cost, wireless data transfer technologies has grown exponentially worldwide, especially the wireless local area networks (WLAN). WLAN's are designed to operate in the 2.4 $\mathrm{GHz}(2.4-2.48 \mathrm{GHz})$ and $5 \mathrm{GHz}$ frequency bands [1]. Home $\mathrm{RF}$ and Bluetooth also operate in the same frequency range, which are free and unlicensed international bands that allow high-speed data communications become more attractive, so long as the user follows the using standard on the channel, can use freely. Since the $2.4 \mathrm{GHz}$ band is frequently used for various applications, moving to the relatively $5 \mathrm{GHz}$ U-NII bands gives 802.11a a significant advantage [2]. For example, The Bluetooth with $802.11 \mathrm{~g}$ specifications is developed by combing $802.11 \mathrm{~b}$ and $802.11 \mathrm{a}$. It has extensively deployed from the Media Access Control (MAC) layer of the WLAN standard, IEEE 802.11b that has already had. IEEE Std 802.11a is provided to support the higher rate physical layer (PHY) for operation in the $2.4 \mathrm{GHz}$ band by adopting OFDM technology with the high transmission speed of 54 Mbps [3, 4]. The performances of dramatically have made them attractive in the related circuit design [5-8].
The primary advantage of direct down-conversion system is that there is no image frequency produced, and consequently much simpler and inexpensive filters can be used [9]. However, direct down-conversion system has disadvantages such as local oscillator (LO) self-mixing, dc-offsets, and other local oscillator (LO) signal leakage at the receiver that can seriously degrade the performance of a receiver through increased noise and inter-modulation direction. To combat this problem, several techniques have been suggested such as the use of a doubly frequency at the output of the LO $[6,7]$ and the use of a sub-harmonic mixer [10-12].

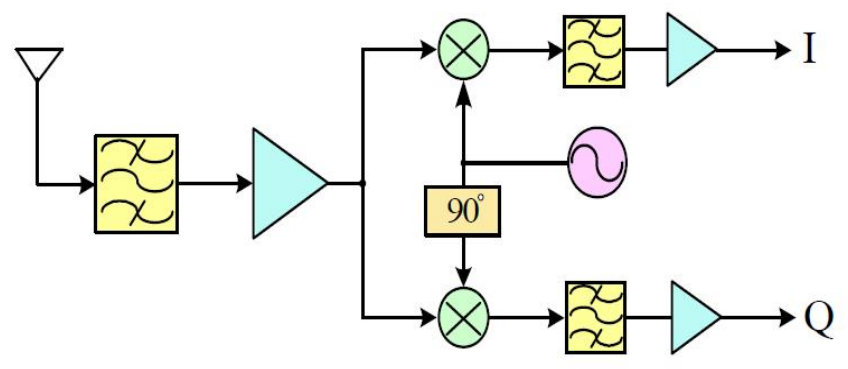

FIGURE I. STRUCTURE OF DIRECT DOWN-CONVERSION SYSTEM.

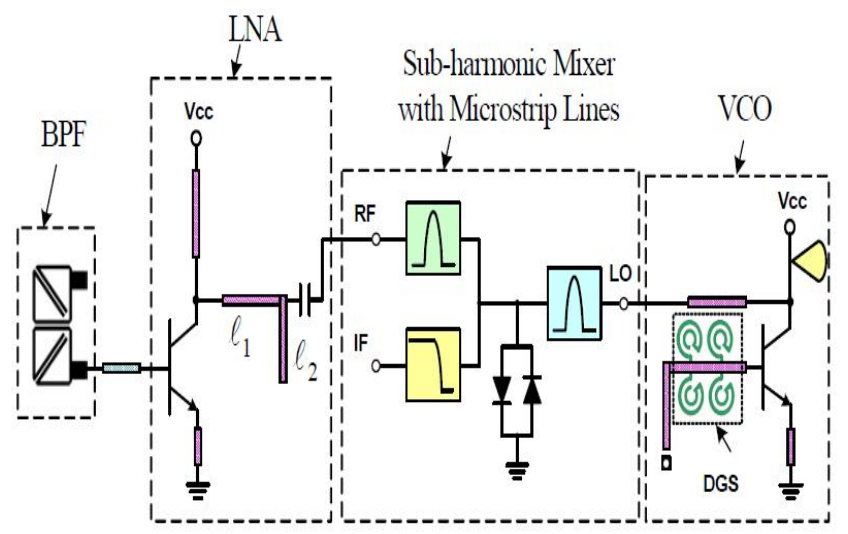

FIGURE II. THE PURPOSED STRUCTURE FOR DIRECT DOWNCONVERSION SYSTEM 


\section{STRUCTURE OF A DiRECT DOWN-CONVERSION SYSTEM USING SUB-HARMONIC MIXER WITH MICROSTRIP LINES}

This paper describes a $2.4 \mathrm{GHz}$ direct down-conversion system for WLAN systems. The structure of direct downconversion system is shown in figure 1; the proposed structure for the direct down-conversion system is shown in figure 2 . The circuit is formed by a miniaturized bandpass filter, highgain and low-noise two-stage LNA, a sub-harmonic mixer, and a voltage-controlled oscillator with defected ground structure.

This paper is mainly further investigated and designed to the direct down-conversion system in order to meet the best performances. Its advantages of direct down-conversion system are described in detail as follows.

$A$. The structure is succinct owing to not include the circuit of intermediate frequency. Moreover, the circuit can reduce the non-linear conversion distortion, can rise the linearly, also can improve the dynamic range of the direct down-conversion system.

$B$. The structure of zero intermediate frequency (ZIF) provides some advantages, namely, it eliminates the need for complex high frequency band pass IF surface acoustic wave (SAW) filters and IF OP, can simplify the structure of the circuit and cheaper charges.

$C$. Because the frequency synthesizer only uses one in the direct down-conversion way, it can reduce unnecessary interference and coupling, and can accelerate the locking time of the frequency synthesizer. Therefore, saving the expensive filter, avoiding the interference of the mirror image frequency, cheaper charges, higher circuit integrity to make the structure of zero intermediate frequency become an attractive design style extremely.

$D$. Direct down-conversion system has the disadvantages of local oscillator (LO) self-mixing and other local oscillator (LO) signal leakage to the antenna. Those signals will interfere to the nearby frequency band of the receiver. The proposed doubly sub-harmonic mixer can reduce the questions easily by raising isolation degree in this paper.

\section{DESIGN OF A DIRECT DOWN-CONVERSION SYSTEM USING SUB-HA RMONIC MIXER WITH MICROSTRIP LINES}

\section{A. A Miniaturized Bandpass Filter}

Because of bandpass filter can reject or attenuate signals out of the operating band to retrieve the signals of being desired. In other words, the passband region of a bandpass filter with good performances are including lower insertion loss, higher return loss, higher flatness, lower ripples wave, sharper roll-off band; however, it is except which can exhibited high insertion loss and low reflection loss on cut-off region. The method for designing filter is usually by using microstrip to realize lumped component circuit. For example, the thin and long microstrip line will be regarded as the inductor effect; open end coupling of microstrip or coupling of two conductors on upper and lower will be regard as capacity effect. When designing the transmission line lumped component model, correct is most important. In other words, those factors are considered, which including parasitic effect, radiation question and $\mathrm{Q}$ values, perhaps will cause much insertion loss. The proposed modeling of resonator with specific wavelength is utilizing the characteristic of transmit ion line cross-coupled in both sides of the passband to produce the desired transmission zero.

It is mainly the concept of using the electric magnetic field cross-phase-coupled to transmit a lot of signals on the many transmission paths of the input and output ends. Let the proposed circuit can produce attenuation pole and smoothly group delay in limited frequency point by the influence of the multipath effect to signals transmission phase.

It is different from the traditional square open-loop resonator, this paper combine the design concepts of electric magnetic field cross-phase-coupled and resonator dual-mode ring resonator, design and implementation a miniaturized narrow band-pass filter, and there are effects applied to receive the necessary signals from the antenna end of the radio frequency front-end.

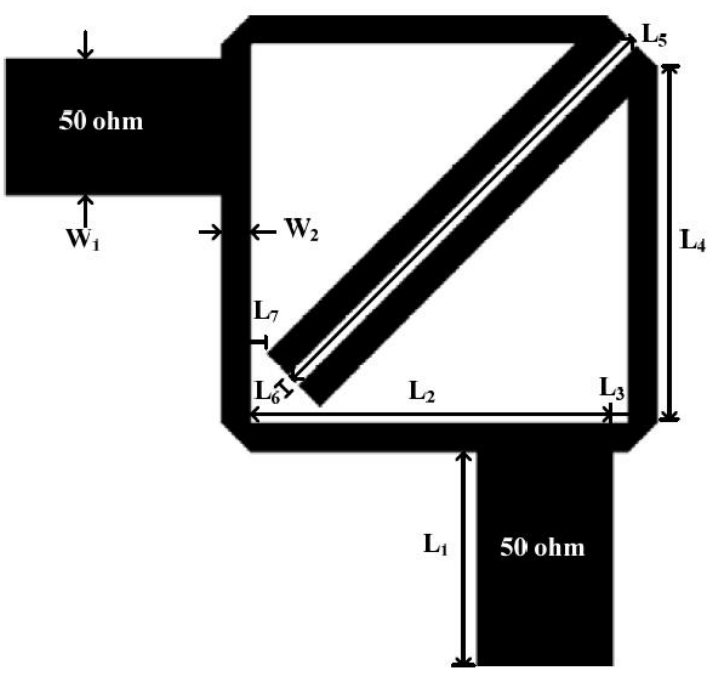

FIGURE III. THE PURPOSED DIAGRAM FOR UNIT RESONATOR.

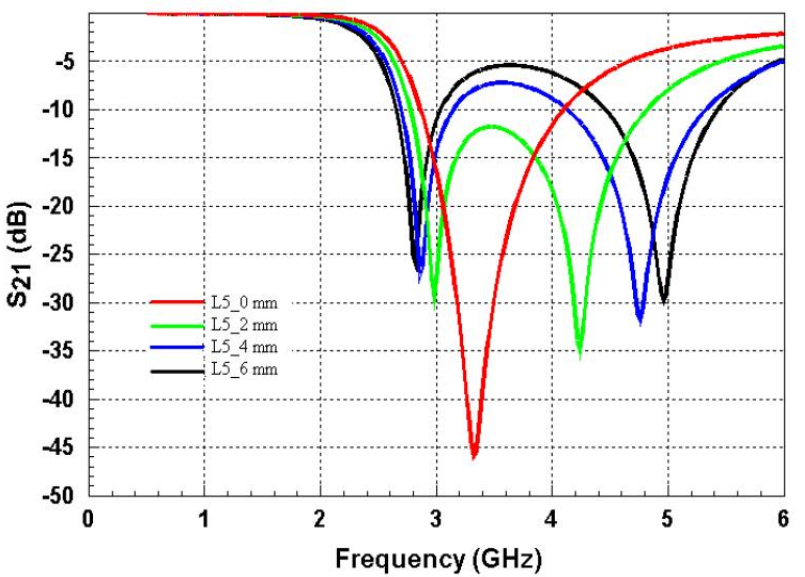

FIGURE IV. SIMULATED FREQUENCY RESPONSES FOR ADJUSTING THE PARAMETER OF L5. 


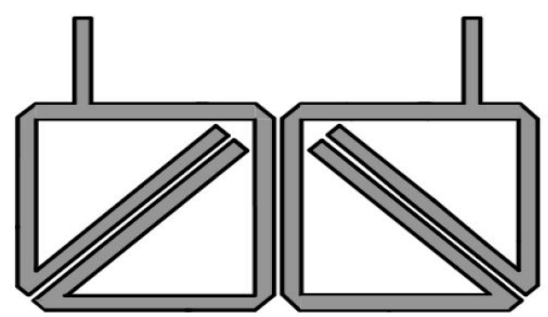

FIGURE V. THE PROPOSED DIAGRAM FOR NARROW B ANDPASS FILTER.

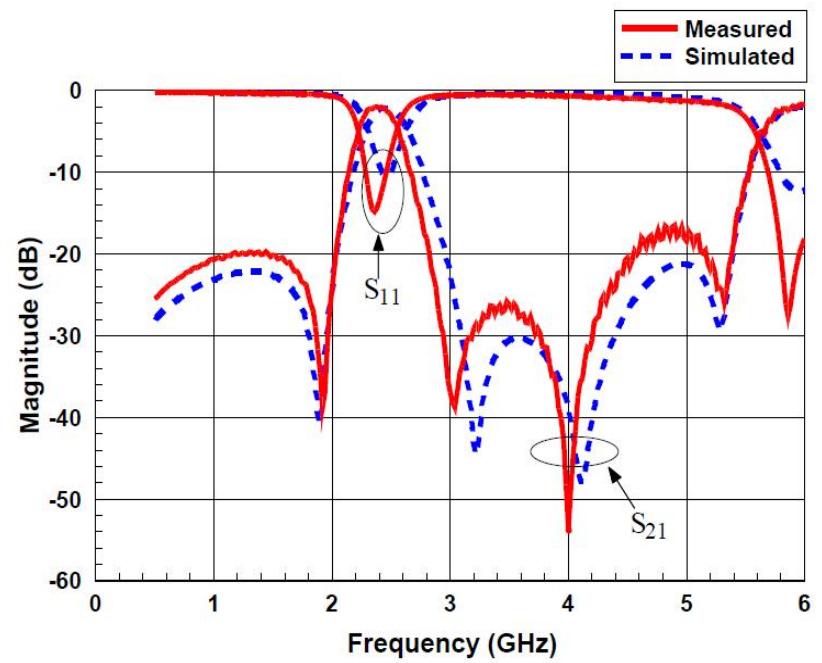

FIGURE VI. THE SIMULATED AND MEASURED RESULTS FOR THE BANDPASS FILTER.

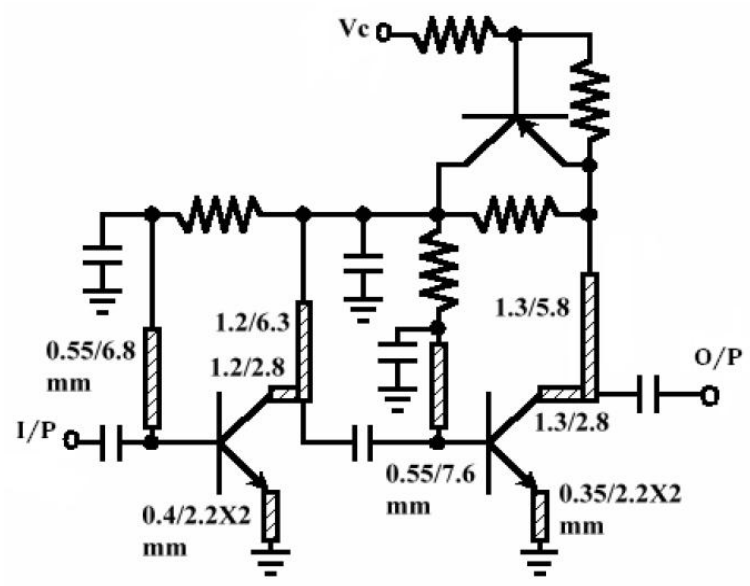

FIGURE VII. A COMPLETE SCHEMATIC OF TWO-STAGE LNA.

The proposed diagram for unit resonator cell is shown in figure 3 , and its each length of unit resonator cell can be arranged in table 1. Figure 4 shows the simulated results of unit resonator cell by using Sonnet EM full-wave simulation software. After increasing the length of L5, the extra transmission zero point can be produced in higher frequency will also be shift up to higher frequency position, and another one is shift down to lower frequency position at same time. The each dimension for adjusting the parameter of L5 in unit resonator is shown in table 2 . The proposed complete structure of miniaturizing magnetic coupling narrow bandpass filter is shown in figure 5; From the simulated results shown in figure 6 , we can find that this circuit has a lot of advantages, which are including a narrow passband with $200 \mathrm{MHz}$, lower insertion loss with $-2.12 \mathrm{~dB}$ @ $2.46 \mathrm{GHz}$, wider suppression bandwidth with $4.46 \mathrm{GHz}$ out-of-band response, and smaller area with $12 \times 6 \mathrm{~mm} 2$. The bandpass filter circuit is fabricated using conventional technology on a FR4 substrate with thickness 0.8 $\mathrm{mm}$.

TABLE I. THE DIMENSIONS OF THE PARAMETERS IN UNIT RESONATOR.

\begin{tabular}{|c|c|c|c|c|c|c|c|c|c|}
\hline Parameters & $\mathrm{W}_{1}$ & $\mathrm{~W}_{2}$ & $\mathrm{~L}_{1}$ & $\mathrm{~L}_{2}$ & $\mathrm{~L}_{3}$ & $\mathrm{~L}_{4}$ & $\mathrm{~L}_{5}$ & $\mathrm{~L}_{6}$ & $\mathrm{~L}_{7}$ \\
\hline Length $(\mathrm{mm})$ & 1.89 & 0.42 & 3 & 5 & 0.2 & 5 & 6.8 & 0.2 & 0.2 \\
\hline
\end{tabular}

TABLE II. THE DIMENSIONS FOR ADJUSTING THE PARAMETER OF L5 IN UNIT RESONATOR.

\begin{tabular}{|c|c|c|c|c|}
\hline $\mathrm{L}_{3}(\mathrm{~mm})$ & 3.6 & 2.6 & 1.6 & 0.6 \\
\hline $\mathrm{L}_{4}(\mathrm{~mm})$ & 8.4 & 7.4 & 6.4 & 5.4 \\
\hline $\mathrm{L}_{5}(\mathrm{~mm})$ & 0 & 2 & 4 & 6 \\
\hline
\end{tabular}

\section{B. A High-gain and Low-noise Two-stage Microstrip LNA}

An ideal LNA should have sufficiently high gain and low noise figure (NF) in order to obtain the high IP3, high linearity, and low power consumption. It is desirable to adopt the singleended input differential output LNA (S-to-D LNA) with sufficiently high gain, low NF, and high linearity. It is focused on high gain and low NF on designing the first stage LNA, the factor of high gain is considered as a key element on designing the second stage LNA. In order to reach the stability, low noise figure and low reflection coefficient of the proposed circuit at the same time, put a microstrip inductance as a negative feedback on the emitter end of the first stage LNA. Thus, not only improving input impedance, reduce the noise figure, but also promoted the stability of the circuit. This paper proposed a two-stage low noise amplifier for $2.4 \mathrm{GHz}$ by using the $\mathrm{SiGe}$ transistor BFP620. Input and output matching networks are needed to reduce undesired reflections and improve the power capabilities [13]. In figure 7, the two-stage low noise amplifier adopts that the way of short stub match, uses the FR4 with relative permittivity of 4.4 and thickness of $0.8 \mathrm{~mm}$, two-stage LNA design the bias DC-current first and match the circuit, and cooperate with Serenade computer-aid simulation software to finished.

For the first stage of LNA, in designing of the input matching network, we shunt a length of short stub $(0.55 / 6.8$ $\mathrm{mm})$ to the base end of the transistor BFP620 and connect a capacity together in series. Similarly, for output matching network, we series a length of short stub $(1.2 / 2.8 \mathrm{~mm}$ and $1.2 / 6.3 \mathrm{~mm}$ ) in L style to the collector end of the transistor BFP620 and series a output capacity together which must be tunabled and matched to $50 \Omega$ [13]. Similarly, we can design the second stage of the LNA networks matching. We shunt a length of short stub $(0.55 / 7.6 \mathrm{~mm})$ to the base end of the transistor BFP620 and connect a capacity together in series. Similarly, for output matching network, we series a length of 
short stub $(1.3 / 2.8 \mathrm{~mm}$ and $1.3 / 5.8 \mathrm{~mm})$ in $\mathrm{L}$ style to the collector end of the transistor BFP620 and series a output capacity together which must be tunabled and matched to $50 \Omega$.

Input and output matching networks are designed for 2.4 $\mathrm{GHz}$ microstrip LNA by utilizing Serenade computer-aid simulation software. The calculations for length of microstrip line for input/output matching network by using smith chart via Serenade computer-aid simulation software is optimum. In the design, from the simulated results shown in figure 8 , we can find the performances of the two-stage low noise amplifier for $2.4 \mathrm{GHz}$ are very good. The matching networks are designed for microstrip LNA having an overall noise figure of $1 \mathrm{~dB}$, the 1-dB compression point $\left(\mathrm{P}_{1 \mathrm{~dB}}\right)$ of $4 \mathrm{dBm}$, the $S_{11}$ and $S_{22}$ are about $-17 \mathrm{~dB}$ and $-25 \mathrm{~dB}$ respectively, a voltage gain of $26 \mathrm{~dB}$, and the $\mathrm{S} 12$ is greater than $30 \mathrm{~dB}$ by using SiGe transistor BFP620 in common emitter configuration.

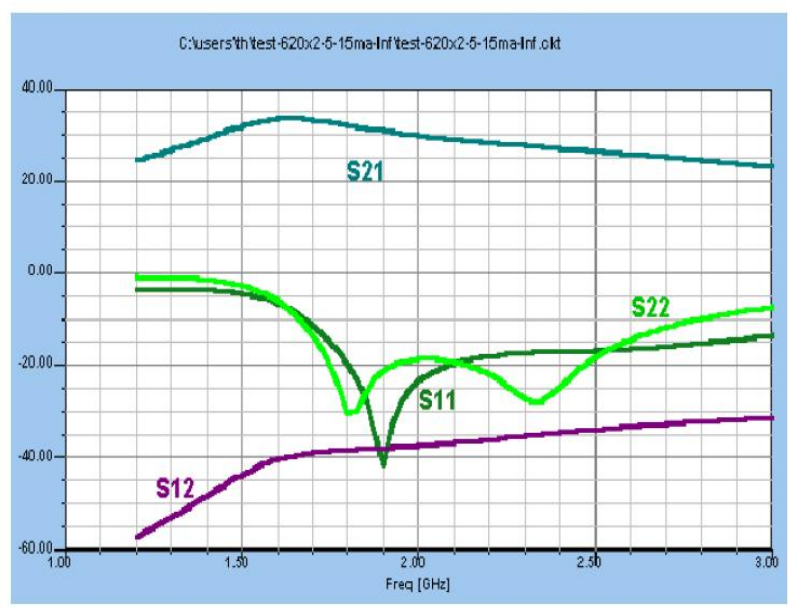

FIGURE VIII. SIMULATED RESULTS FOR S PARAMETERS OF THE TWO-STAGE LNA.

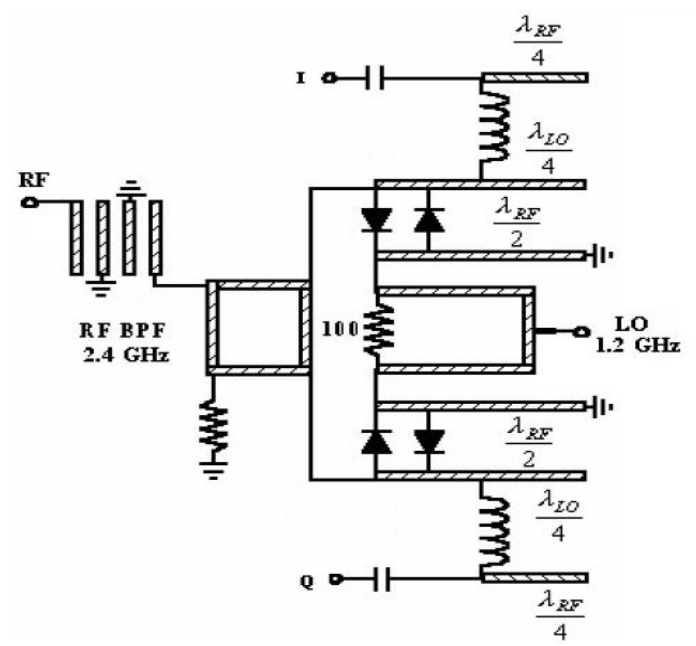

FIGURE IX. SCHEMATIC DIAGRAM OF DOUBLY MIXER.

\section{A Sub-harmonic Mixer with Microstrip Lines}

The sub-harmonic mixers are very useful at microwave wave communication system due to its directly down frequency from radio frequency (RF) to zero intermediate frequency (IF), low local oscillator (LO) frequency and low cost merits.

At designing mixer, those are must considered performances including conversion loss, the isolation level between RF and LO ports, 3rd-order intercept point (IP3) and 1 -dB compression point etc. A metric of 3rd-order intercept point products is important for evaluating device performance particularly in amplifiers or mixers whether have perfect linearity. $\mathrm{IP}_{3}$ and $\mathrm{P}_{1 \mathrm{~dB}}$ are an important parameter for amplifiers or mixers designers to estimate spurious free dynamic range (SFDR).

In the structure finished designing, when fed the LO signal at frequency $\mathrm{f}$ with large input power, it will to produce a radio frequency signal at twice $f$ frequency in RF end by the nonlinear characteristic of the diode. IF input here LO signal and radio frequency signal at the same time, then basic wave $f$ and odd times harmonic waves ( $\mathrm{n} f, \mathrm{n}=1,3,5, \ldots$ ) will be suppressed, and it will output the even harmonic waves ( $\mathrm{f}, \mathrm{n}$ $=2,4,6, \ldots)$, this characteristic mainly makes the best of the characteristic that different electronic length of microstrip line will produce open or short circuit in different frequency.

Figure 9 is the structure diagram of doubly sub-harmonic mixer by the way of direct conversion. From figure 9, we can see that the whole structure consists of BPF, branch coupler, Wilkinson divider, and two pair of anti-parallel diodes which is Schottky-barrier diode of HSMS-2800. The anti-symmetric diodes are mainly providing harmonic mixing to derive larger doubly harmonic signals on single diode mixing by its nonlinear characteristic of $\mathrm{I}-\mathrm{V}$ curve on single balance characteristic. In other word, this diode has merits of high operation frequency, low capacitance and low conversation loss, so that is excellent using for the SHM design.

The mixer is intended to operate for RF frequency of 2.4 $\mathrm{GHz}$ transforming to IF frequency of $10 \mathrm{MHz}$, so that the frequency of $\mathrm{LO}$ oscillator is $1.2 \mathrm{GHz}$. Besides, in figure 9, the operation of the receiver is as follow: The received RF signal will be amplified by the LNA. Then the signal will pass through the band selector RF bandpass filter to remove the unwanted signal or interference signal. After that the signal will pass through the $90^{\circ}$ branch coupler and output two RF signals which the phase difference is $90^{\circ}$. Then the signal passes through the simple LC high-pass filter. The objective of highpass filter is to increase the isolation between RF ports and LO port. After that these two signals will be sent into mixer and mix with in-phase LO oscillation signal. Because we also utilized the same phase through Wilkinson divider to divide the LO signal into two to supply required LO signal of the two SHM. The RF signal can be converted directly t to baseband signal.

A $\lambda_{\mathrm{RF}} / 2$ at RF frequency short stub circuit located on the LO side of the diode pair, such that the RF signal is terminated with a virtual ground but the LO signal is not affected. The use of a short stub also provide dc and IF return path to ground. Similarly, A $\lambda_{\mathrm{LO}} / 4$ at LO frequency open stub circuit located on the RF side of the diode pair, such that the LO signal is terminated with a virtual ground but the RF signal is not affected. Moreover, a shunt $\lambda_{\mathrm{RF}} / 4$ at RF frequency open stub is located between IF port and low-pass filter. This stub 
transmission line prevents RF signal leakage to IF port (RF chock) but the IF signal can still be extracted from the top side of the anti-diode pair.

Figure 10 shows the simulated results of doubly subharmonic mixer for $S$ parameter by utilizing Serenade computer-aid simulation software. It has not included Q channel circuit in the simulated circuit, and because I channel circuit is the same as $\mathrm{Q}$ channel circuit, only, namely the simulated results for $\mathrm{S}$ parameters of doubly mixer only in I channel including bandpass filter, $90^{\circ}$ branch coupler and Wilkinson divider. To obtain the characteristics of the proposed sub-harmonic mixer, the circuit as figure 9 had implemented and measured on the FR4-dielectric substrate with the same parameters of the implemented filter circuit mentioned above.

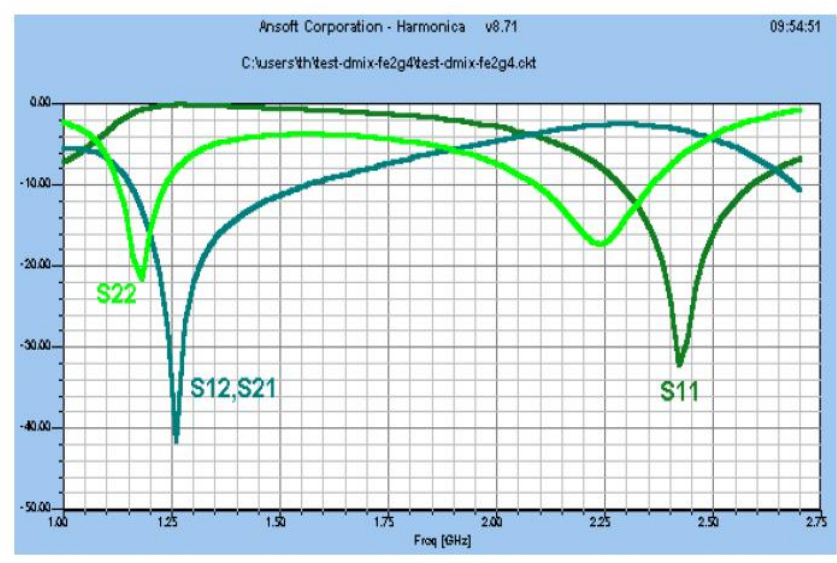

FIGURE X. SIMULATED RESULTS FOR S PARAMETERS OF DOUBLY MIXER IN I CHANNEL.

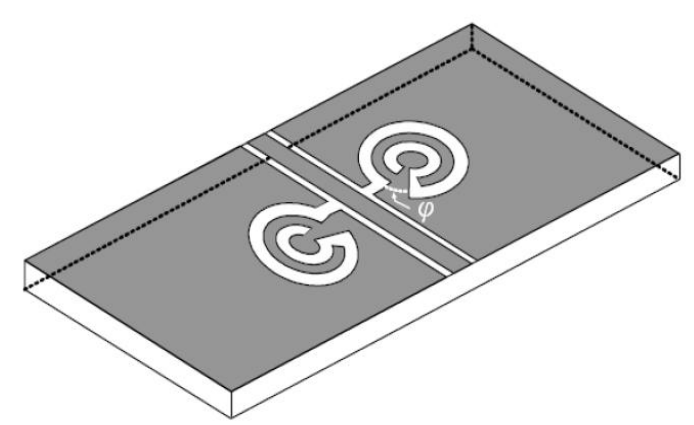

FIGURE XI. THE PROPOSED STRUCTURE OF HIGH Q CIRCUIT SPIRAL-SHAPED DGS.

\section{A Voltage-controlled Oscillator Using Defected Ground Structure}

A microwave oscillator presented in this work using a spiral-shaped DGS structure with a high-Q factor provides high power, high DC to RF conversion ratio, and low phase noise. With the DGS structure implemented in the ground plane, the advantage of slow-wave characteristics can be obtained to reduce the length of microstrips. However, the disadvantage of dielectric resonators cannot integrating to microwave integrated circuits (MMIC) can be improved. The DGS dimension barely affects the dimension of the microstrip line located on the top of the substrate when applied to oscillators. Since the Q factor of the resonator determines the phase noise dominantly, most of working has been performed by people focusing on the increase of $Q$ factor of the resonator $[14,15]$.

The proposed spiral-shaped DGS structure with a high-Q factor is depicted in figure 11. Can learn that etch the specific shape on the ground from references [16, 17], can change the route where electric magnetic field and electric current are distributed, and then result a transmission zero point at being needed frequency. However, with the difference of DGS shape, the $\mathrm{Q}$ value of transmission zero point will be different. It will depend on distribution situation of its electric magnetic field. For understanding the steep difference of transmitting zero point by using DGS in the list of references, we simulate this high Q value spiral-shaped DGS and other two kinds of form which applied to surface wave guide DGS, the comparison of their simulation results is shown in Fig. 12. Which curve (3) is most typical DGS [16] among them, curve (2) is DGS [17] of L type, the curve (1) is designed style of the spiral DGS. The simulated results exhibit that the spiral-shaped DGS is provided with supreme $Q$ value among them.

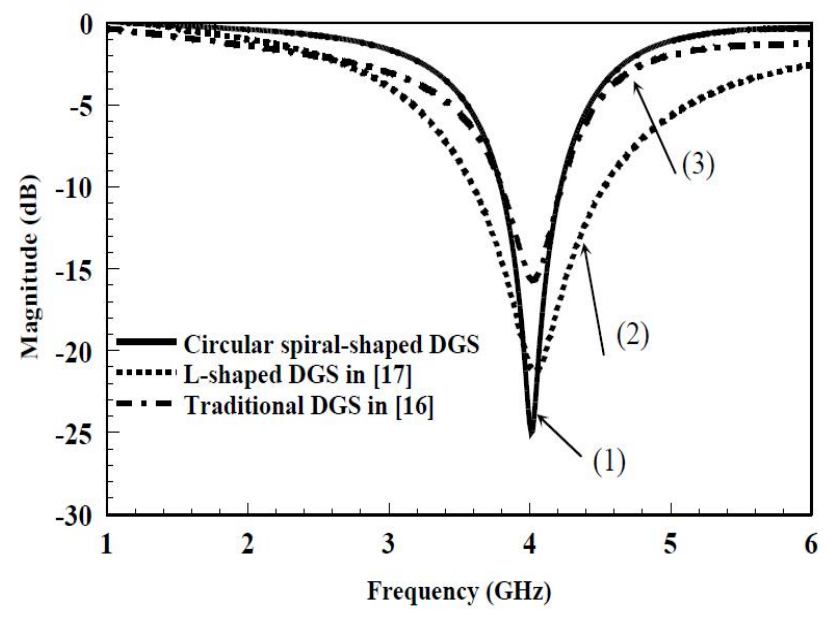

FIGURE XII. COMPARISONS BETWEEN CONVENTIONAL DGS STRUCTURE AND HIGH Q CIRCUIT SPIRAL-SHAPED DGS.

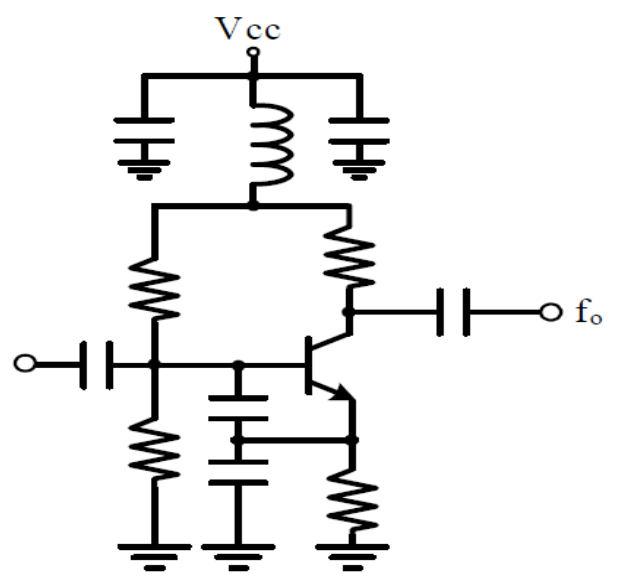

FIGURE XIII. THE COMPLETED CIRCUIT OF VCO WITH NEGATIVE RESISTANCE 
TABLE III. THE SIMULATED AND MEASURED RESULTS OF THE BANDPASS FILTER.

\begin{tabular}{|c|c|c|c|c|}
\hline Parameters & $\mathrm{BW}_{3 \mathrm{~dB}}$ & $\begin{array}{c}<20 \mathrm{~dB} \\
\text { Suppression }\end{array}$ & Insertion loss & Return loss \\
\hline Simulation & $0.2 \mathrm{GHz}$ & $4.46 \mathrm{GHz}$ & $\begin{array}{c}-2.12 \mathrm{~dB} @ \\
2.46 \mathrm{GHz}\end{array}$ & $\begin{array}{c}-10.48 \mathrm{~dB} @ \\
2.46 \mathrm{GHz}\end{array}$ \\
\hline Measurement & $0.22 \mathrm{GHz}$ & $3.7 \mathrm{GHz}$ & $\begin{array}{c}-2 \mathrm{~dB} @ 2.38 \\
\mathrm{GHz}\end{array}$ & $\begin{array}{c}-14.82 \mathrm{~dB} @ \\
2.36 \mathrm{GHz}\end{array}$ \\
\hline
\end{tabular}

TABLE IV. RELATION BETWEEN ANGLESФAND FREQUENCIES. (UNIT: GHZ)

\begin{tabular}{||c|c|c|c|c||}
\hline \hline & $\mathrm{f}_{\mathrm{o}}$ & $\mathrm{f}_{\mathrm{c}}$ & $\mathrm{f}_{\mathrm{n} 1}$ & $\mathrm{f}_{\mathrm{n} 2}$ \\
\hline$\varphi=35^{\circ}$ & 6.0 & 4.5 & 4.0 & 9.18 \\
\hline$\varphi=45^{\circ}$ & 6.28 & 4.74 & 4.16 & 9.7 \\
\hline$\varphi=60^{\circ}$ & 6.62 & 4.96 & 4.36 & 10.2 \\
\hline$\varphi=90^{\circ}$ & 7.66 & 5.43 & 4.75 & 11.26 \\
\hline
\end{tabular}

For a more in-depth study of the resonance frequency characteristics of the spiral-shaped DGS, we change the size of the angle $\varphi$, and observe their relationship with resonance frequency as shown in table 4 . Which $\mathrm{f}_{\mathrm{c}}$ is 3 - $\mathrm{dB}$ cut-off frequency point, $f_{o}$ is the fundamental frequency, $f_{n 1}$ and $f_{n 2}$, respectively are for the first and second notch frequency. From table 6 , we can find when the greater the angle $\varphi$ of circular spiral-shaped DGS will caused the transmission zeros of the upper frequencies, conversely, when the smaller the angle $\varphi$ of circular spiral-shaped DGS will caused the transmission zeros of the lower frequencies. Use the characteristic of the high $\mathrm{Q}$ value and the concept of input impedance of transmission lines, we design a DGS structure to improve phase noise oscillator.

Oscillator is comprised by three basic units, which are active components, terminating network and load network. Resonant circuit is usually designed as dielectric resonator in the past. But in view of the future of highly integrated RF microwave circuit development, therefore the paper completed the function of the resonance by planar spiral-shaped DGS, not only can avoid losing original properties of inductive and capacitive in different frequency due to use lumped components, but also its character of high-Q may significantly reduce the phase noise at the load network. As a result of the consumption of passive components for resonance circuit, in order to maintain the oscillator constant oscillation, active components must continue to provide energy by high negative resistance circuit.

In figure 13 is the configuration of the proposed negative resistance circuit. In order to design a stable VCO circuits, we must take inductors and capacitors by series and parallel resonant circuits respectively into equivalent circuit to get greater values of negative resistance.

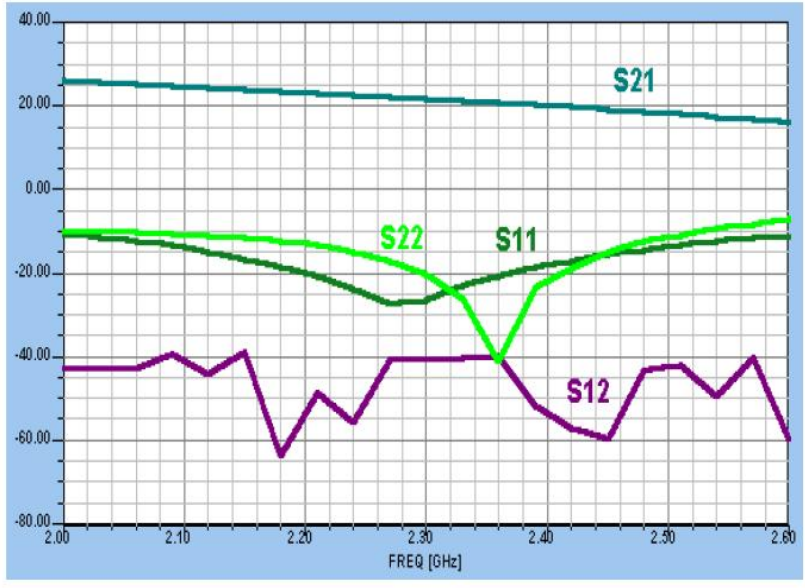

FIGURE XIV. MEASURED RESULTS FOR S PARAMETERS OF THE TWO-STAGE LNA.

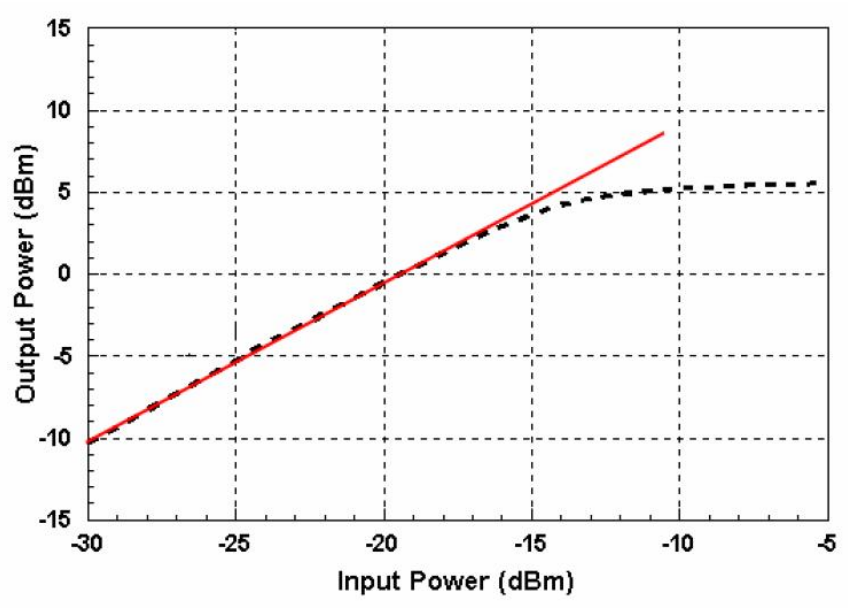

FIGURE XV. MEASURED RESULTS FOR P1DB OF THE TWO-STAGE LNA.

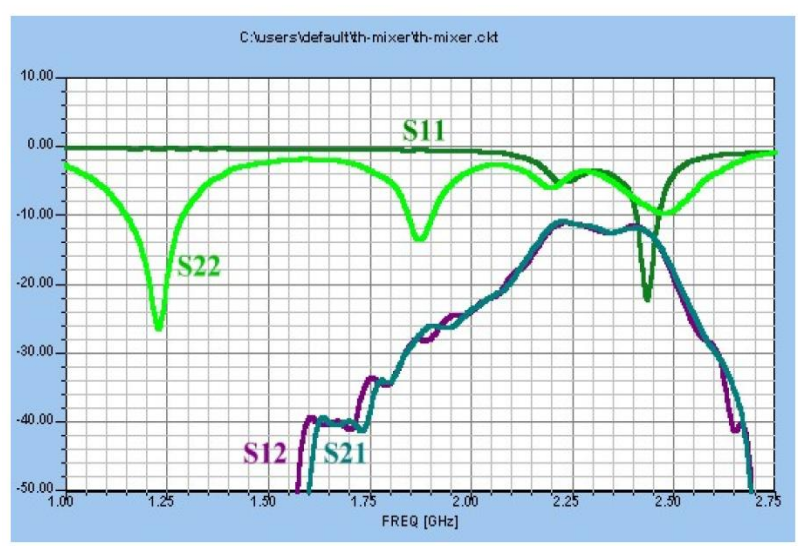

FIGURE XVI. MEASURED RESULTS FOR S PARAMETERS OF DOUBLY MIXER IN I CHANNEL. 


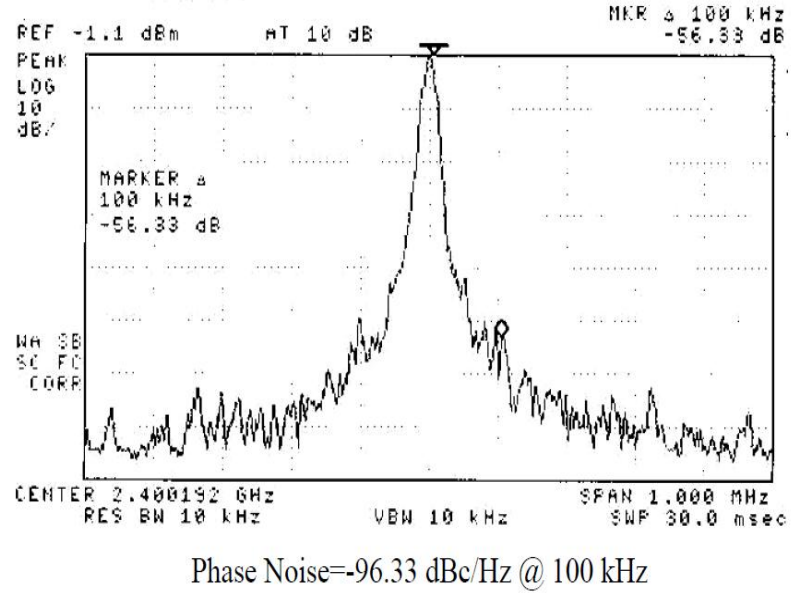

FIGURE XVII. MEASURED RESULTS FOR PHASE NOISE OF VCO.

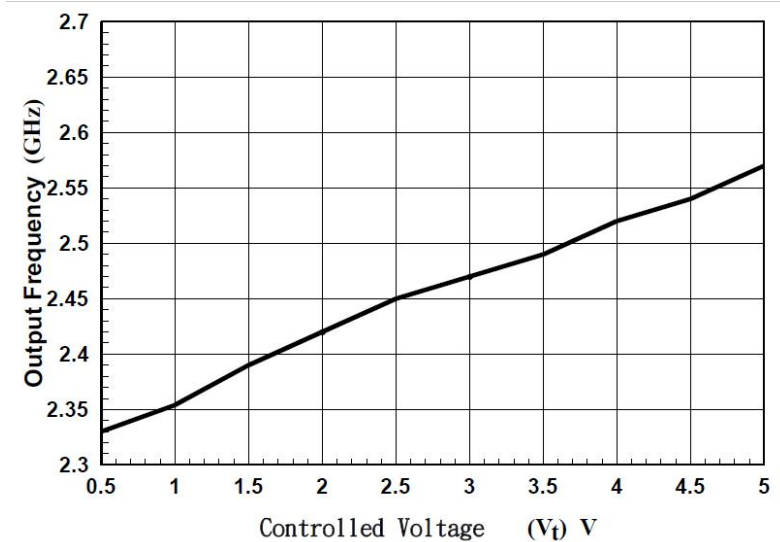

FIGURE XVIII. MEASURED RESULTS FOR GAIN FACTOR OF VCO.

\section{The Measured Results and Discussion The MEASURED RESUlTS AND DISCUSSION}

\section{A. A Miniaturized Bandpass Filter}

Figure 6 shows the simulated and measured results of $S_{11}$ and $S_{21}$ for the bandpass filter, and its records of the entire data are shown in table 3 . From the measured results, we can find that this circuit has a lot of advantages, which are including a narrow passband with $220 \mathrm{MHz}$, lower insertion loss with -2 $\mathrm{dB} @ 2.38 \mathrm{GHz}$, wider suppression bandwidth with $3.7 \mathrm{GHz}$ out-of-band response, and smaller area with $12 \times 6 \mathrm{~mm}^{2}$.

\section{B. A High-gain and Low-noise two-stage Microstrip LNA}

In the LNA design, from the measured results shown in figure 14, we can find the performances of the two-stage low noise amplifier for $2.4 \mathrm{GHz}$ are very well. The matching networks are designed for microstrip LNA operating from a 2.4-V supply and having an overall noise figure of $15 \mathrm{~dB}$, the $1-\mathrm{dB}$ compression point $\left(\mathrm{P}_{1 \mathrm{~dB}}\right)$ of $4 \mathrm{dBm}$, the $S_{11}$ and $S_{22}$ are about $-17.5 \mathrm{~dB}$ and $-21.5 \mathrm{~dB}$ respectively, a voltage gain of 24 $\mathrm{dB}$, and the $\mathrm{S}_{12}$ is greater than $40 \mathrm{~dB}$ by using SiGe transistor BFP620 in common emitter configuration. The simulated and measured results of the two-stage LNA are shown in table 5, and we can find the simulated and measured results are matching very well.

\section{A Sub-harmonic Mixer with Microstrip Lines}

When the input power at LO port of the doubly mixer is 8 $\mathrm{dBm}$, the measured results for $\mathrm{S}$ parameters of doubly mixer in I channel are shown in figure 16. The value of input reflection loss $\mathrm{S}_{11}$ at $2.438 \mathrm{GHz}$ is about $-22.5 \mathrm{~dB}$. The value of input reflection loss $\mathrm{S}_{22}$ at $1.21 \mathrm{GHz}$ is about $-24 \mathrm{~dB}$. When the input power at LO port of the doubly mixer is $5 \mathrm{dBm}$, the value of conversion loss at $2.43 \mathrm{GHz}$ is about $14.53 \mathrm{~dB}$.

As the basic schematic layout of the fabricated SHM is shown in figure 9, and the measured performances are summarized in table 6. From the experimental results, it shows that the value of the $\mathrm{IIP}_{3}$ is about $16 \mathrm{dBm}$ when the input power of the $\mathrm{P}_{1 \mathrm{~dB}}$ is about $6 \mathrm{dBm}$. With the description for LNA above, we can find the output values of $\mathrm{OIP}_{3}$ and $\mathrm{P}_{1 \mathrm{~dB}}$ for LNA lower than the input values of IIP $_{3}$ and $\mathrm{P}_{1 \mathrm{~dB}}$ for mixer respectively; therefore, it will not make the mixer produce the phenomenon of saturating. We can also calculate the input value of $\mathrm{P}_{1 \mathrm{~dB}}$ is about $-16.2 \mathrm{dBm}$, the input value of $\mathrm{IIP}_{3}$ is about $-7.2 \mathrm{dBm}$, the noise figure is about $1.4 \mathrm{~dB}$; Can know by this data, the dynamic range of this front circuit is the same as LNA, thedynamic range characteristic with broadness.

TABLE V. SIMULATED AND MEASURED RESULTS OF THE TWOSTAGE LNA.

\begin{tabular}{|c|c|c|}
\hline \multirow{2}{*}{ Parameter } & \multicolumn{2}{|c|}{ Vcc $=2.4 \mathrm{~V}, @ \mathrm{f}_{\mathrm{o}}=2.4 \mathrm{GHz}$} \\
\cline { 2 - 3 } Results & simulation & measurement \\
\hline $\mathrm{NF}$ & $1 \mathrm{~dB}$ & $1.4 \mathrm{~dB}$ \\
\hline $\mathrm{S}_{11}$ & $-17 \mathrm{~dB}$ & $-17.5 \mathrm{~dB}$ \\
\hline $\mathrm{S}_{22}$ & $-25 \mathrm{~dB}$ & $-21.15 \mathrm{~dB}$ \\
\hline $\mathrm{S}_{21}$ & $26 \mathrm{~dB}$ & $21.25 \mathrm{~dB}$ \\
\hline $\mathrm{P}_{1 \mathrm{~dB}}$ & $4 \mathrm{dBm}$ & $4 \mathrm{dBm}$ \\
\hline OIP3 & - & $13.18 \mathrm{dBm}$ \\
\hline Reverse-Isolation & $36 \mathrm{~dB}$ & $40 \mathrm{~dB}$ \\
\hline
\end{tabular}

TABLE VI. MEASURED RESULTS OF SUB-HARMONIC MIXER.

\begin{tabular}{|c|c|}
\hline \multicolumn{2}{|c|}{ SUB-HARMONIC MIXER @ Plo=8 DBM (DOWN-CONVERSION) } \\
\hline $\mathrm{S}_{\mathrm{RF}}$ & $-22.5 \mathrm{~dB} @ 2.43 \mathrm{GHz}$ \\
\hline $\mathrm{S}_{\mathrm{LO}}$ & $-24 \mathrm{~dB} @ 1.21 \mathrm{GHz}$ \\
\hline LO isolation & $>60 \mathrm{~dB} @ 1.2 \mathrm{GHz}$ \\
\hline Conversion Loss & $14.53 \mathrm{~dB} @ 2.43 \mathrm{GHz}$ \\
\hline $\mathrm{P}_{1 \mathrm{~dB}}$ & $6 \mathrm{dBm} @ 2.43 \mathrm{GHz}$ \\
\hline $\mathrm{IIP}_{3}$ & $16 \mathrm{dBm} @ 2.43 \mathrm{GHz}$ \\
\hline
\end{tabular}

D. A Voltage-controlled Oscillator Using Defected Ground Structure

The high Q-factor of the spiral-shaped DGS provides high carrier output power and low phase noise. The output power is obtained as $14.2 \mathrm{dBm}$ at $2.438 \mathrm{GHz}$ with $2.0 \mathrm{~V} \mathrm{DC}$ supply and $24 \mathrm{~mA}$ current consumption. It can be seen that the $\mathrm{Q}$ factor of spiral-shaped DGS is 14 , the phase noise is observed about -96 
$\mathrm{dBc} / \mathrm{Hz}$ at $10 \mathrm{kHz}$ offset and $\mathrm{DC}$ to $\mathrm{RF}$ conversion ratio is calculated $52.8 \%$. The microwave oscillator using a DGS is fabricated on FR4 substrate. A considerable enhanced output power was produced using the spiral-shaped DGS developed here.

\section{CONCLUSIONS}

In this paper, we have succeeded to applying a subharmonic mixer with microstrip line to have a zero intermediate frequency system, we also utilized a high $\mathrm{Q}$ circuit spiral-shaped DGS at voltage controlled oscillator to successfully suppress the spurious noise; To reduce the unwanted signals, we proposed a narrow bandpass filter by a series of resonators; The LO-RF and LO-IF isolations of subharmonic mixer are greater than $60 \mathrm{~dB}$ and the conversion loss is $14.53 \mathrm{~dB}$ at $2.43 \mathrm{GHz}$; The phase noise of VCO with spiralshaped DGS can be $-96.6 \mathrm{~dB}$ at $10 \mathrm{kHz}$; The $\mathrm{S}_{21}, \mathrm{~S}_{11}$, and $\mathrm{S}_{22}$ of LNA are $21.25 \mathrm{~dB},-17.25 \mathrm{~dB}$, and $-21.25 \mathrm{~dB}$, respectively. The complete circuits of a direct down-conversion system using sub-harmonic mixer with microstrip lines can be work well at $2.4 \mathrm{GHz}$ band.

\section{REFERENCES}

[1] D. D. Krishna, M. Gopikrishna, C. K. Anandan, P. Mohanan, and K., Vasudevan, "CPW-Fed Koch Fractal Slot Antenna for WLAN/WiMAX Applications," IEEE Antennas and Propag. Lett., vol. 7,pp. 389-392, Nov. 2008.

[2] G. C.-Y. Chen, K. K.-M. Chan, K. Rambabu, "Miniaturized yagi class of antennas for GSM, WLAN, and WiMax applications," IEEE Transactions on Consumer Electronics - IEEE TRANS CONSUM ELECTRON, vol. 56, no. 3, pp. 1235-1240, 2010.

[3] SIG, Bluetooth Specification Version 1.0B, 1999.

[4] IEEE Standard 802.11b: Higher-Speed Physical Layer Extension in the $2.4 \mathrm{GHz}$ Band.

[5] Sung-Mao Wu, Yen-Ting Lu, Ming-Hui Huang and Kao-Yi Wang, "Using Multilayer S-Type Coupled Resonators to Realize the 2.4 G Bandpass Filters", 2010 Asia-Pacific Microwave Conference (APMC),Yokohama, Japan, pp. 1197-1200, Nov. 2010.

[6] Jiang Xiaolin; Gu Xuemai; Li Haijun, "Based on 2.4G Wireless Communications System Design", Networks Security Wireless Communications and Trusted Computing (NSWCTC), 2010 Second International Conference on, vol. 2, pp. 553 - 556, 24-25 April 2010.

[7] Eun-Pyo Hong; Yong-Seok Hwang; Hyung-Joun Yoo, “A Folded RF Front-end with Low Flicker Noise for Bluetooth and ZigBee application", Microwave Conference, 2007. APMC 2007. Asia-Pacific, pp. 1-4, 11-14 Dec. 2007.

[8] Joong Han Yoon, In Gi Jeong, Sung Jae Ha, Young Chul Rhee, “Design of a microstrip-fed monopole antenna with a rectangular slit ground and a rectangular projection strips for dual-band WLAN operations", Microwave Conference Proceedings (APMC). 2011 Asia-Pacific, pp. 721-724, 5-8 Dec. 2011.

[9] Brad R. Jackson and Carlos E. Saavedra, "A CMOS Subharmonic Mixer with Input and Output Active Baluns", Microwave and Optical Technology Letters. vol. 48, no. 12, pp. 2472-2478, December 2006.

[10] L. Sheng, J. C. Jensen, and L. E. Larson, "A wide-bandwidth Si/SiGe HBT direct conversion sub-harmonic mixer/down converter," IEEE $J$. Solid-State Circuits, vol. 35, no. 9, pp. 1329-1337, Sept. 2000.

[11] R. Svitek and S. Raman, "5-6 GHz SiGe active I/Q subharmonic mixer with power supply noise effect characterization," IEEE Trans. Microw. Wireless Compon. Lett., vol. 14, no. 7, pp. 319-321, Jul. 2004.

[12] K. J. Jon, M. Y. Park, C. S. Kim, and H. K. Yu, " Subharmonically pumped CMOS frequency conversion (up and down) circuits for 2-GHz WCDMA direct-conversion transceiver," IEEE J. Solid-State Circuits, vol. 39 , no. 6 , pp. 871-884, Jun. 2004.
[13] Kumar, D.; Verma, R.; Singhal, N.; Bhaskar, L.; "Performance analysis of microstrip line matching network for $2.4 \mathrm{GHz}$ microwave LNA via Matlab programming," Antennas \& Propagation Conference, LAPC 2009. Loughborough, pp.705-708, 16-17 Nov. 2009.

[14] P. G. Wilson and R. D. Carver, "An easy-to-use FET DRO design procedure suited to most Cad program," IEEE MTT-S Diges, pp. 10331036, 1989.

[15] K. Hosoya, S. Tananka, Y. amamiya, T. Niwa, H. Shimawaki, and K. Honjo, "A low-phase 38-GHz HBT MMIC oscillator utilizing a novel transmission line resonator," IEEE MTT-S Diges, pp. 47-50, 2000.

[16] Y. Q. Fu, G. H. Zhang, and N. C. Yuan, "A Novel PBG Coplanar Waveguide," IEEE Micro- wave and Wireless Components Letters, vol.11, no.11, pp.447-449, Nov. 2001.

[17] M. Kim and B. Lee, "Novel L-Shaped DGS for Coplanar Waveguide," in Asia-Pacific Micro- wave Conf., Nov. 2003. 